\title{
The Phenomena of Sudden Change in Hypersonic Inlets Self-Starting
}

\author{
Capability \\ Yinan JIA ${ }^{1}$, Lianjie YUE ${ }^{1}$ and Xinyu CHANG ${ }^{1}$ \\ ${ }^{1}$ Institute of Mechanics, Chinese Academy of Sciences, No.15 Beisihuanxi Road, Beijing 100190, \\ China \\ Corresponding Author: yuelj@imech.ac.cn
}

\begin{abstract}
Wind tunnel experiments and numerical calculations were conducted to investigate the influence of several key factors on self-starting capability. The changes of maximum ICR with cowl shock strength, boundary layer thickness and wall temperature were explored and the sudden change of inlet self-starting capability was observed during a certain range of these influencing factors. A transitional phenomenon was obtained in both experiments and numerical simulations. It is found that the sudden changes of inlet self-starting capability with boundary layer thickness and wall temperature take place with a wide domain of transitional phenomenon.
\end{abstract}

\section{Nomenclature}

$\alpha=$ cowl angle

$\mathrm{H} \quad=$ cowl height

$\mathrm{L}=$ the length of bottom plate upstream cowl section

$\mathrm{p}_{\infty}=$ static pressure of incoming flow

$\mathrm{p}_{0} \quad=\quad$ total pressure of incoming flow

$\mathrm{Ma}_{\infty}=$ Mach number of incoming flow

$\mathrm{T}_{0}=$ total temperature of incoming flow

$\operatorname{Re}_{\infty, \mathrm{m}}=$ unit Reynolds number of incoming flow

\section{I . Introduction}


As an important component of scramjet, the hypersonic inlet must operate in a started mode to capture and compress a certain amount of air for processing by remainder of the engine. Thus self-starting ability is a very important parameter of hypersonic inlet, that means when the factors resulting inlet unstart are removed the inlet could be restarted. Kantrowitz proposed a theoretical model for supersonic inlet restart with assumption of a normal shock wave in front of the internal contraction and quasi-steady one-dimensional isentropic internal flow with sonic condition at the inlet throat ${ }^{[1,2]}$. But unfortunately the restart flow pattern for hypersonic inlet is much different from the assumption and there is a strong shock associated with a large scale boundary layer separation ${ }^{[3]}$. Many experiments have verified that the inlet can restart at internal contraction ratio beyond Kantrowitz limit ${ }^{[3-5]}$.

Goldberg studied various parameters that affected self-starting capability of a twodimensional inlet with thick boundary layer and took the Schlieren images of flow patterns ${ }^{[6]}$. He found that the inlets started with separation which could cause much shock losses with full mass flow capture, thus he introduced the total pressure recovery as the key parameter dominating whether an inlet can restart, and cold wall could help the inlet restart. Van Wie investigated the starting characteristics of a small-scale rectangular inlet at Mach 3 condition ${ }^{[7]}$. He classified the inlet unstart profile into two broad categories as either "hard" or "soft" according to a series of experimental results. These research not only focus on the flow patterns inside the inlet during the restart process but also pays attention to the affecting factors of inlet starting capability.

Inlet self-starting characteristics have been extensively studied in the past decades, but it is still not well understood. The influencing factors always coupled during the research and there is still a lack of particular analysis about each individual factor as well as the connection between them. In this paper, experiments and numerical simulations on decoupling influencing factors are conducted to investigate self-starting capability. Based on a series of inlet self-starting experiments, the effect of cowl shock strength and boundary layer thickness on the inlet performance is observed. Meanwhile, the influence of wall temperature is studied by using numerical calculation. The maximum inlet self-starting internal contraction ratio (ICR) is obtained.

\section{Test Facility}

The experiments were conducted in the GJF shock tunnel of the Institute of Mechanics, Chinese Academy of Science. The shock tunnel is equipped with an 11.2 $\mathrm{m}$ long driver section and a $22 \mathrm{~m}$ long driven section filled with compressed air, downstream which is the axisymmetric nozzle and vacuum experimental tank, as shown 
in Figure 1. For these experiments, the nozzle Mach number $\mathrm{M}_{\infty}$ was 4.0. The total pressure $\mathrm{P}_{0}$ and total temperature $\mathrm{T}_{0}$ of the air were $13.8 \mathrm{MPa}$ and $430 \mathrm{~K}$, and the unit Reynolds number $\operatorname{Re}_{\infty}, \mathrm{m}$ was $1.18 \times 10^{7} / \mathrm{m}$. The valid experimental duration is about 40 ms.

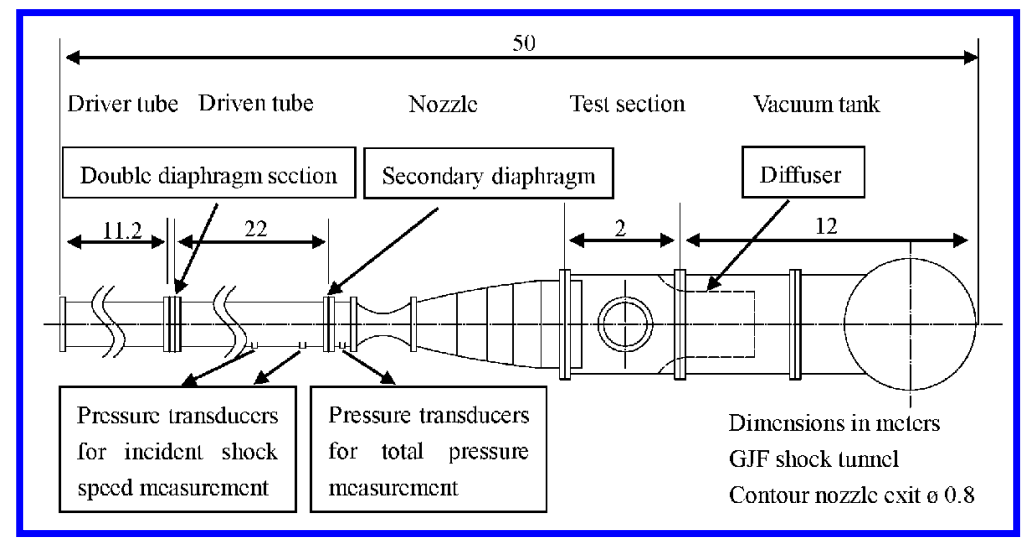

Figure 1 Sketch of the shock tunnel

A novel test method is proposed for surveying the hypersonic inlet self-starting capability within the short running time of the shock tunnel ${ }^{[8]}$. A set of equipment are designed to accomplish the inlet self-starting experiments ${ }^{[8]}$, as shown in Figure 2. A Terylene diaphragm is placed at the inlet exit with a coherent electric resistance wire on its leeward insulated from the shock tunnel and inlet model. During the initial experimental stage, the inlet would be choked into big buzz due to the obstruction of the diaphragm. Concurrently, the total pressure of the shock tunnel starts the delay-time signal generator, which in turn outputs a pulse voltage signal after a few milliseconds to trigger the high-voltage igniter. The diaphragm is then ruptured by the heating effect of the electric resistance wire. Note that, the delay time is pre-set so that the diaphragm rupture will occur after the unstart flow of the inlet reaches a quasi-steady state. As with that in conventional wind tunnels, the restarting flow process of the inlet can be therefore investigated to check its self-starting capability after the choke disappears.

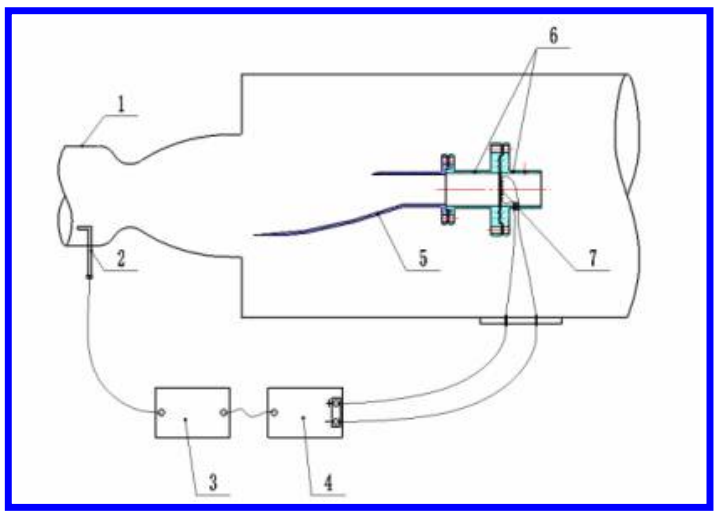

1 Shock tunnel nozzle 2 Pressure probe 3 Delay time signal generator 4 Electrical igniter

5 Hypersonic inlet 6 Device for diaphragm installation 7 Terylene diaphragm 


\section{Experimental Model}

The simplified inlet model is shown in Figure 3 and Figure 4. The model has a constant span width of $80 \mathrm{~mm}$. Three parameters cowl angle $\alpha$, cowl height $\mathrm{H}$ and the bottom plate length upstream the cowl L could be changed in the model design. The cowl angle $\alpha$ related to the cowl shock strength. Five angles were used in the experiments, that were $7^{\circ}, 8^{\circ}, 9^{\circ}, 11^{\circ}$ and $15^{\circ}$, respectively. L determined the boundary layer thickness at the cowl section. The longer was L, the thicker was the boundary layer. $\mathrm{H}$ measured the cowl height and was set as $20,30,40 \mathrm{~mm}$. L was chosen as 50 , $100,200,300,400,500,600 \mathrm{~mm}$. In our research, the maximum ICR capturing inlet self-start was used to characterize the inlet self-starting capability. A set of cowl plates with different throat height were designed to experimentally obtain the maximum ICR.

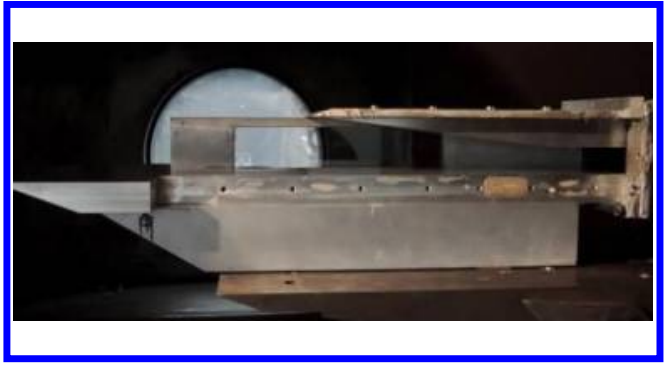

Figure 3 Photo of the inlet model

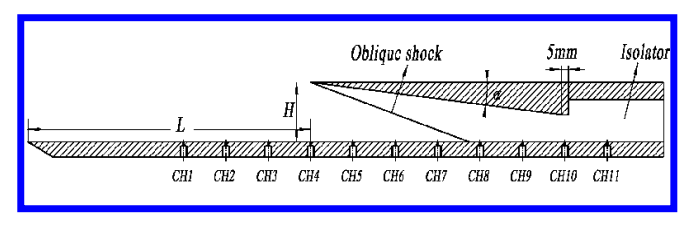

Figure 4 Sketch of the inlet model

The inlet model was also equipped with 11 static pressure ports along the centerline of the bottom wall with an interval distance of $20 \mathrm{~mm}$ to monitor the location of separation bubble as well as the starting state of the inlet. Dynamic pressure transducers, marked $\mathrm{CH} 1-\mathrm{CH} 11$ (CH4 port always located at $10 \mathrm{~mm}$ upstream the cowl station and CH5 $20 \mathrm{~mm}$ downstream the cowl), were installed directly on the model wall so that the internal cavum and the conduit do not degrade the actual frequency response significantly. The transducers have a range of $200 \mathrm{kPa}$ for $\mathrm{CH} 1-\mathrm{CH} 8$ and $500 \mathrm{kPa}$ for $\mathrm{CH} 9-\mathrm{CH} 11$, with an accuracy of $0.3 \%$ in the full range and the natural response frequency of $30 \mathrm{kHz}$. A 128-channels data acquisition system, consisting of voltage signal amplifiers and the National Instruments DAQ PXI-6150 cards, was used for pressure measurement with the sampling frequency of $31.2 \mathrm{kHz}$ per channel.

Two windows with silica glasses were installed in the sidewalls for optical access to visualize the internal flow field. During the experiments, Schlieren images were taken to observe the unstart/restart flow field. The frame frequency was set 5000/s, and the camera would record 500 images which covered the valid test time of the wind tunnel. 


\section{Numerical Description}

To assist the investigation of the influence of wall temperature on inlet self-starting capability, numerical simulations were performed for the experimental models. The full $\mathrm{N}-\mathrm{S}$ equations for two-dimensional turbulent flow were solved by the finite volume method. The turbulence model of k- $\omega$ SST was employed to close the governing equations. The numerical flux through each cell face was evaluated using a secondorder total variation diminishing (TVD) scheme based on an approximate Riemann solver named Harten-Lax-van Leer contact (HLLC). The minmod limiter was used to suppress spurious oscillations near the discontinuities, while high-order accuracy was retained away from the jumps. A second-order fully implicit scheme was employed to discretize the time terms. In addition, methods of multigrid and dual time-step were used to accelerate the convergence. Our previous studies ${ }^{[9,10]}$ have shown that the solver can efficiently resolve high Mach number flows.

\section{Results and Discussion}

\section{a. The influence of cowl angle on inlet self-starting capability}

Figure 5 shows the variation of ICR limit with cowl angle for different height $\mathrm{H}$ and ratio $\mathrm{L} / \mathrm{H}$. Inlet self-starting maximum ICR decreases as the cowl angle increases. The cowl shock wave, interacting with the boundary layer, determines the size of the separation bubble upstream the throat. With stronger shock wave, the separation bubble expands and may cause the flow choke at the throat. Thus relatively larger throat area, which means larger aerodynamic throat, is required to assure that the separation bubble can get through. Note that there is a sudden change when the cowl angle varies from $7^{\circ}$ to $9^{\circ}$ which means the hypersonic inlet is sensitive to cowl angle in this range. When cowl lip angle changes from $9^{\circ}$ to $15^{\circ}$, the maximum ICR decreases smoothly.

For the inlet model with cowl angle of $9^{\circ}$, the separation bubble is speculated large enough to induce an aerodynamic throat taking place of geometrical throat to limit the maximum flux that can get through the inlet. While for the small cowl angle of $7^{\circ}$, the separation bubble is not large enough to unstart the inlet. The inlet model can restart until the ICR increases to an extent at which the geometrical throat choke causes the inlet to unstart. Thus, the sudden change in Figure 5 is generated. 


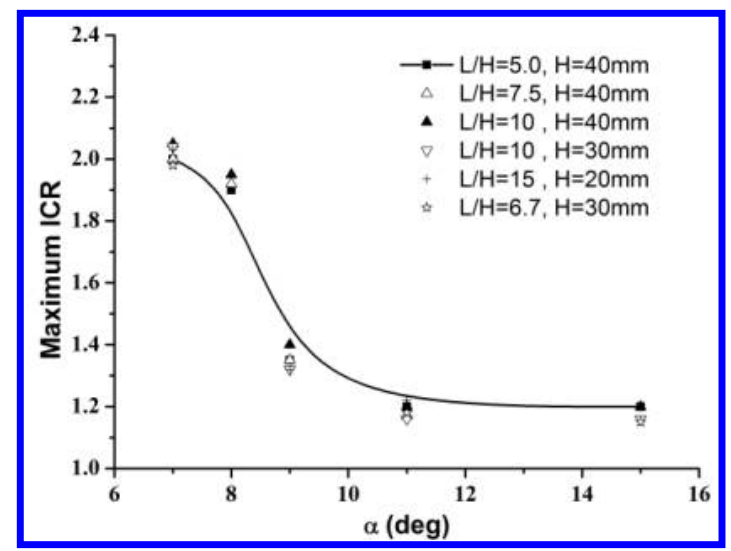

Figure 5 Variation of ICR limit with cowl angle

\section{b. The influence of boundary layer thickness on inlet self-starting capability}

$\mathrm{L}$ determines the boundary layer thickness at the cowl lip section and $\mathrm{H}$ measures the cowl lip height. The ratio of $\mathrm{L}$ to $\mathrm{H}$ can represent the relative thickness of boundary layer at cowl lip section. For cases with $\alpha=11^{\circ}$, as $\mathrm{L} / \mathrm{H}$ changes, the maximum ICR varies in small extent around 1.2. Meanwhile, for cases with $\alpha=7^{\circ}$, when $\mathrm{L} / \mathrm{H}$ varies from 0 to 15 , the maximum ICR stays near 2.0. However, the inlet self-starting maximum ICR decreases suddenly as L/H increases from 15 to 20, as shown in Figure 6. For large cowl angle models, the maximum ICR stays a small value due to the impact of strong cowl shock wave which enhances the size of separation bubble. For small cowl angle models, the influence of cowl shock wave is relatively negligible and the effect of boundary layer starts to emerge. The influence of boundary layer thickness has a limit: when the relative thickness exceeds this limit, the shock-boundary layer interaction at cowl lip section will be enormously impacted by the thickness of boundary layer, otherwise the relative boundary layer thickness has weak effect on the inlet self-starting capability.

Experimental boundary layer thickness $\delta$ can be estimated by schlieren imagery of the density variation ${ }^{[11]}$. Figure 7 illustrates the boundary layer's variation with length L. The light amplitude variations are related to the density gradient, which is also proportional to the gradient in static temperature through the ideal gas law. By identifying the thickness of the thermal boundary layer from the Schlieren images, an estimate of the velocity boundary-layer thickness is obtained by the combination of the thermal boundary layer thickness with the Prandtl number. Figure 8 shows the variation of velocity boundary layer thickness with L. In this way, the influencing limit of relative boundary layer thickness for $7^{\circ}$ models can be obtained, that is, when $H / \delta>2.9$, the boundary layer thickness has an obvious effect on inlet self-starting capability. 


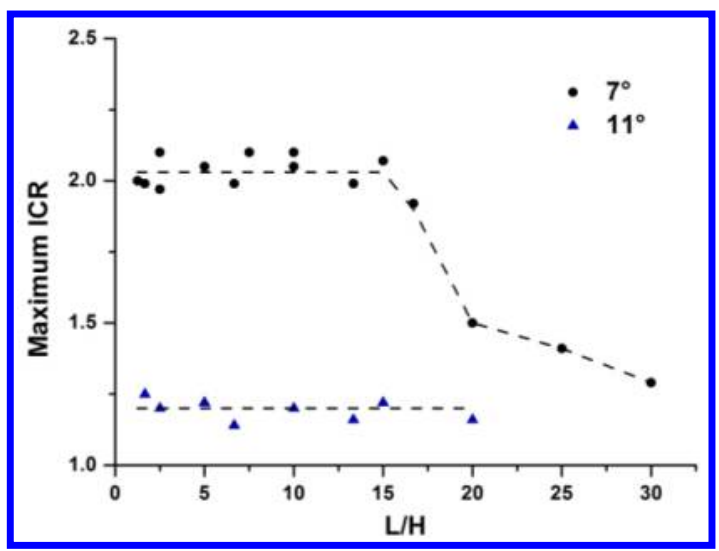

Figure 6 Variation of ICR limit with $\mathrm{L} / \mathrm{H}\left(\alpha=7^{\circ}, 1^{\circ}\right)$

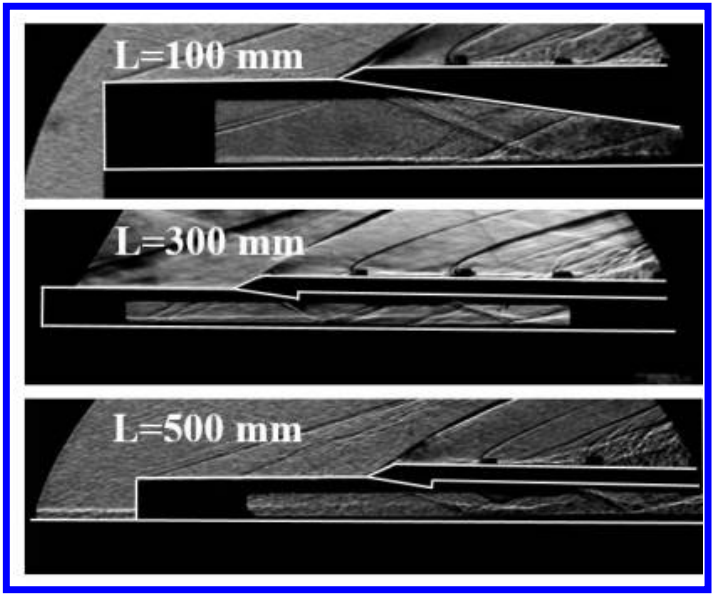

Figure 7 Schlieren images for different $L$

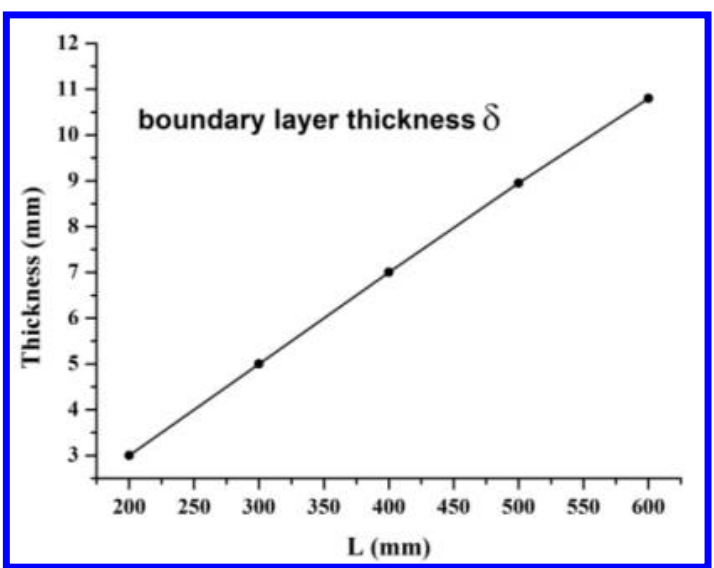

Figure 8 The variation of boundary layer thickness with $L$

During the self-starting process, the choke firstly occurs with a large separation at the inlet cowl section before the diaphragm ruptures. As the downstream pressure reduces, the separation bubble flows downstream. Whether the separation goes through the throat determines the inlets start or unstart. However, a transitional phenomenon was observed in the experiments, in which the separation stayed steadily in the internal section but the separation shock impinged on the cowl. Typical Schlieren images are shown in the Figure 9. From the flow pattern during inlet self-starting process, a separation bubble exists in the internal section and does not disappear. Figure 10 further 
shows the pressure variation at the cowl section $(\mathrm{CH} 4)$ during the inlet unstart/restart process. Before the diaphragm ruptures, big buzz occurs before cowl. As the diaphragm ruptures at $30 \mathrm{~ms}$, the expansion waves propagate upstream. Once the inlet can self-start, the pressure will fall to the same level with the inlet start condition. While during the transitional and unstart process, the pressure at the cowl section still keeps high but oscillates with small amplitude, which illustrates the separation bubble locates near the cowl lip, and oscillates to achieve a dynamic equilibrium. As the separation bubble induces a shock and increases the shock loss in the transitional condition, the total pressure recovery decreases and the inlet cannot obtain enough efficient air to work in the combustor.
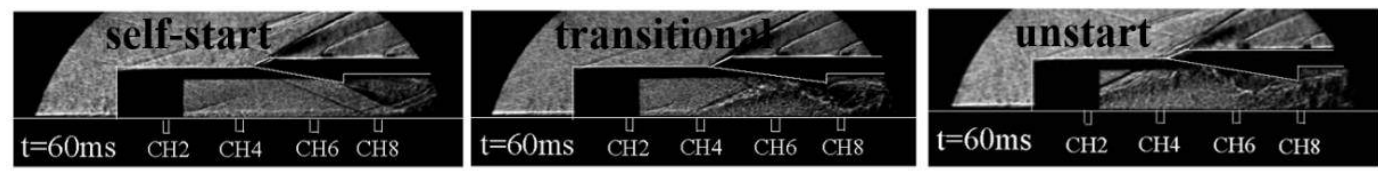

Figure 9 Schlieren images of inlet self-start, unstart and transitional conditions

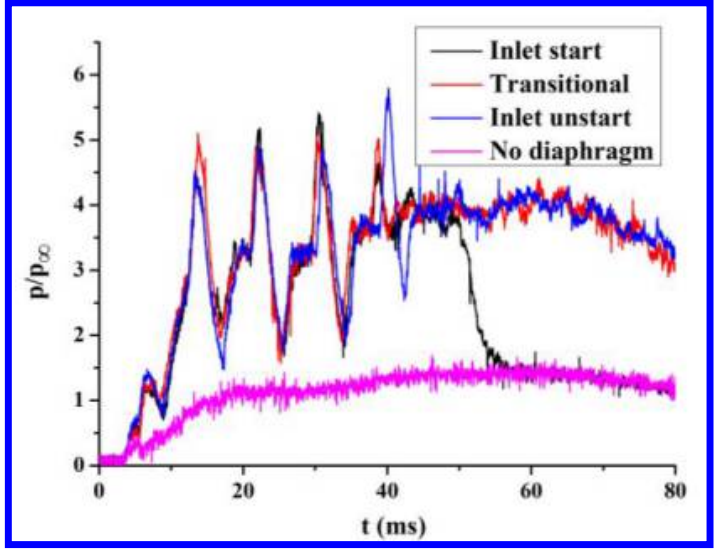

Figure 10 Static pressure-time histories for different conditions at $\mathrm{CH} 4$

When $\mathrm{L} / \mathrm{H}$ raises to 20 , the maximum ICR drops to 1.5 . Transitional phenomenon occurs within a wide range of ICR between 1.5 to 2.1 . When ICR is larger than 2.1, the flow patterns in the inlet is unstart. And when ICR is smaller than 1.5, the inlet can selfstart. The wide range of transitional phenomenon exists while $\mathrm{L} / \mathrm{H}$ is larger than 20 where the self-starting maximum ICR is quite small. And the upper boundary of the transitional ICR range remains near 2.0 which indicates that if the transitional conditions can be transformed to self-starting conditions, the sudden drop of inlet selfstarting capability with $\mathrm{L} / \mathrm{H}$ can be avoided.

\section{c. The influence of wall temperature on inlet self-starting capability}

The impact of cooling wall was surveyed numerically in this work. The computational domain and structured mesh used in the present simulations are shown in Figure 11. The incoming flow conditions are set the same as those in wind tunnel experiments, that is, incoming Mach number of 4.0, the total pressure of $1.4 \mathrm{MPa}$, and 
the total temperature of $430 \mathrm{~K}$. Various wall temperature is set, including adiabatic wall condition. Figure 12 illustrates the variation of inlet restarting maximum ICR with wall temperature. When the wall condition is set as adiabatic, the wall temperature is about $393 \mathrm{~K}$ and the corresponding maximum ICR is 1.2. As wall temperature drops, the selfstarting maximum ICR first jumps to 1.8 at wall temperature of $350 \mathrm{~K}$ and then increases slowly to 2.0 when wall temperature is $100 \mathrm{~K}$. The self-starting capability improves with a decrease in wall temperature because cooling wall can reduce the boundary layer thickness and promote the total pressure recovery coefficient. This tendency is consistent with Goldberg's study ${ }^{[12]}$. But the sudden change of ICR with wall temperature has never been observed. It reveals that the inlet restart capability is very sensitive to wall temperature in this temperature domain, which means a little temperature decrease can result in a high develop of inlet self-starting capability.

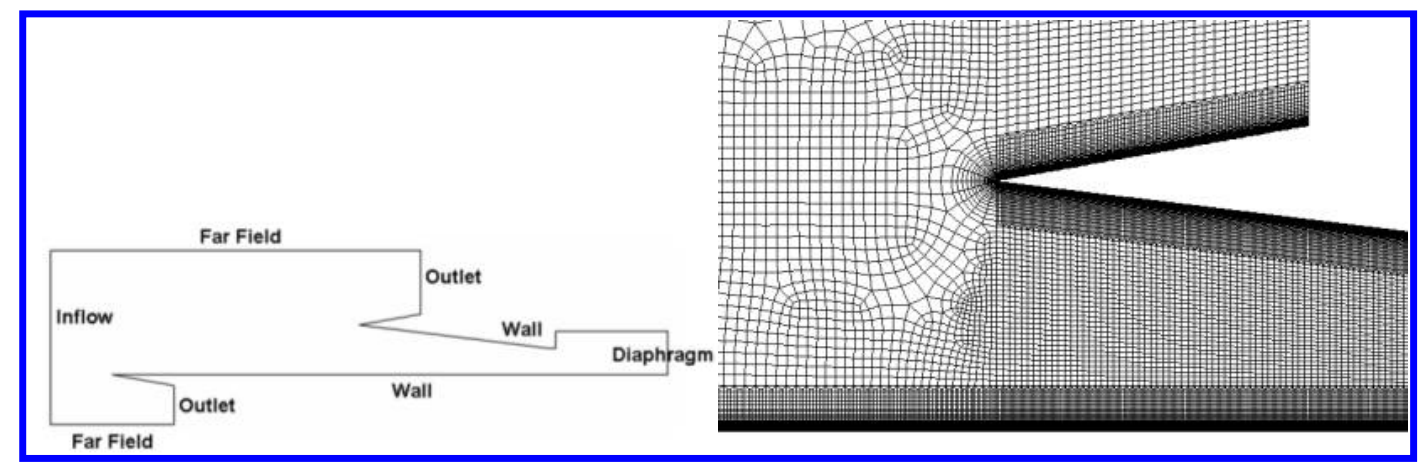

a) Computational domain and boundary conditions

b) Structured mesh

Fig. 11 Computational domain, mesh grids and boundary conditions for the simulation of hypersonic inlets.

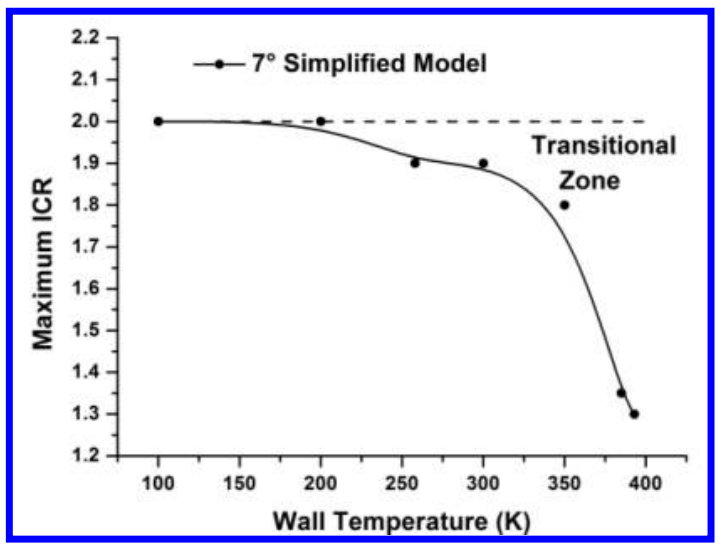

Figure 12 Variation of ICR limit with wall temperature $\left(\alpha=7^{\circ}, \mathrm{L} / \mathrm{H}=10\right)$

The sudden change of inlet self-start maximum ICR occurs with the appearance of wide range of transitional phenomenon as well. For all the calculation cases, the transitional condition take place between self-start maximum ICR to ICR of 2.0, as shown in Figure 12, which indicates transitional condition can be forced into self- 
starting condition by cooling wall. Thus, the sudden drop of inlet self-starting capability with $\mathrm{L} / \mathrm{H}$ can be eliminated.

In both the experiments and the calculations, the upper boundary of transitional phenomenon, namely, the boundary between transitional condition and unstart condition, always stays near the same ICR, respectively. The discrepancy between these two flow patterns should be specified. Figure 13 illustrates the self-starting condition, transitional condition and unstart condition of inlet self-starting process. For transitional condition, the separation bubble induces an aerodynamic throat instead of the geometrical throat in which the flow is supersonic and all the flow flux can get through the inlet. While for unstart condition, the flow chokes at the geometrical throat where the flow velocity is smaller than local sound velocity. Thus, transitional phenomenon is caused by the separation bubble stays in the contraction section while the cause of inlet unstart is the flow choke at geometrical throat which do not alter with wall temperature. Therefore, the upper boundary of transitional zone changes slightly with wall temperature.

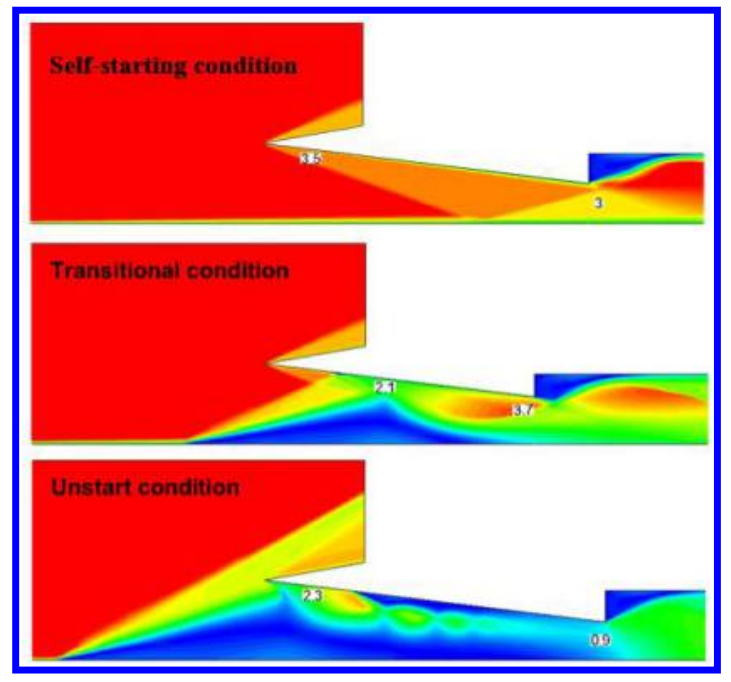

Figure 13 Mach contours of three conditions of inlet restart process $\left(\alpha=7^{\circ}, \mathrm{L} / \mathrm{H}=10\right)$

Figure 14 shows the linear change of boundary layer thickness with wall temperature and no sharp change appears. Thus, the saltation in Figure 12 is little related to the boundary layer thickness variation. Its appearance should be associated with transitional phenomenon, in which a separation bubble is located in contraction section. Unfortunately, the mechanism of transitional mode is still unclear, and needs further investigation. 


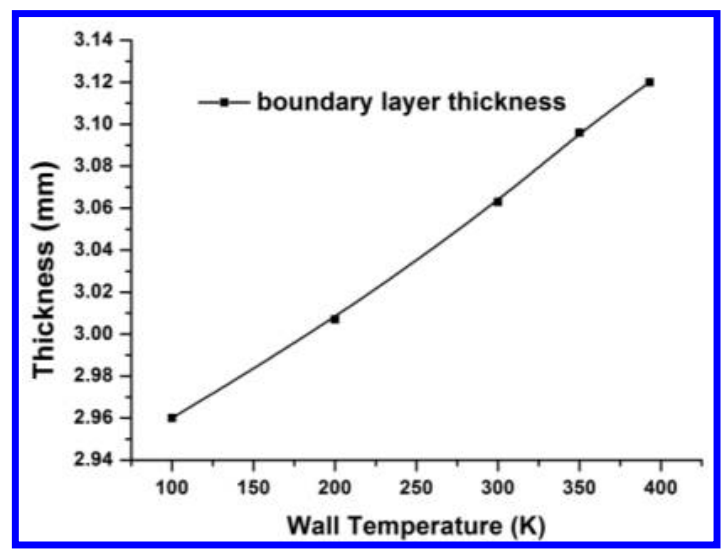

Figure 14 The variation of boundary layer thickness with wall temperature

\section{Conclusions}

There are sudden changes during the variation of inlet maximum ICR with cowl shock strength, boundary layer thickness and wall temperature. For the sudden change with cowl shock strength, the cause is speculated that the strong shock wave inducing aerodynamic throat takes place of geometrical throat to control the maximum flux the inlet can breathe in. The influence of boundary layer thickness starts to emerge when the cowl angle is small. Because the influence of boundary layer thickness is negligible on large cowl angle models with strong shock wave. The influence of boundary layer thickness has a limit: when the relative thickness exceeds this limit, the shock-boundary layer interaction at cowl lip section will be enormously impacted by the thickness of boundary layer, otherwise the relative boundary layer thickness has weak effect on the inlet self-starting capability. For the cases of boundary layer thickness and wall temperature effecting inlet self-starting capability, the sudden change occurs with a wide range of transitional phenomenon. Cooling wall can transform the transitional condition induced by thick boundary layer thickness to self-starting condition. Thus, the reduction of inlet self-starting capability caused by thick boundary layer thickness can be avoided. Meanwhile, the sudden change induced by wall temperature is in close relevance with the movement of separation bubble. But the reason this phenomenon is still unknown.

\section{References}

[1] Kantrowitz, A., and Donaldson, C. Preliminary Investigation of Supersonic Diffusers. NACA ACR-L5D20, May 1945.

[2] Kantrowitz, A. The Formation and Stability of Normal Shock Waves in Channel Flows. NACA TN 1225, March 1947. 
-[3] Bo Sun, Kun-yuan Zhang. Empirical Equation for Self-Starting Limit of Supersonic Inlets. Journal of Propulsion and Power, Vol.26, No.4, 2010, pp. 874-875.

-[4] Veillard, X., Tahir, R.B., Timofeev, E.V., and Molder, S. Limiting Contractions for Starting Simple Ramp-type Scramjet Intakes with Overboard Spillage. Journal of Propulsion and Power, Vol.24, No.5, 2008, pp. 1042-1049.

[5] Molder, S., Timofeev, E.V., and Tahir, R.B. Flow Starting in High Compression Hypersonic Air Inlets by Mass Spillage. AIAA Paper 2004-4130.

[6] Tbeodore, J. Goldberg, Jerry, N. Hefner. Starting Phenomena for Hypersonic Inlet with Thick Turbulent Boundary Layers at Mach 6. NASA TN D-6280, August 1971.

[7] Van Wie, D.M., Ault, D.A. Internal Flowfield Characteristics of a Two-Dimensional Scramjet Inlet at Mach 10. AIAA Paper 1965-088.

[8] Hong LIU, Lianjie YUE, Yabin XIAO, Hui PENG, Xinyu ZHANG. Experimental Method for Hypersonic Inlet Self-starting in Shock Tunnel. AJCPP 2012-173.

[9] Lu, H. B., Yue, L. J., and Chang, X. Y., "Flow Characteristics of Hypersonic Inlets with Different Cowl-Lip Blunting Methods," Science China: Physics, Mechanics and Astronomy, Vol.57, No.4, 2014, pp. 741-752.

doi: 10.1007/s11433-013-5285-0

-[10] Lu, H. B., Yue, L. J., Xiao, Y. B., and Zhang, X. Y., "Interaction of Isentropic Compression Waves with a Bow Shock," AIAA Journal, Vol.51, No.10, 2013, pp. 2474-2484.

doi: 10.2514/1.J052373

-[11] Fotia, M. L., and Driscoll, J. F., "Isolator-Combustor Interactions in a Direct-Connect RamjetScramjet Experiment”, Journal of Propulsion and Power, Vol. 28, No.1, 2012, pp. 83-95. doi: 10.2514/1.B34367

[12] Tbeodore, J. Goldberg, Jerry, N. Hefner. Starting Phenomena for Hypersonic Inlet with Thick Turbulent Boundary Layers at Mach 6. NASA TN D-6280, August 1971. 